\title{
Sensitivity of Wave Field Evolution and Manifold Stability in Chaotic Systems
}

\author{
Nicholas R. Cerruti and Steven Tomsovic \\ Department of Physics, Washington State University, Pullman, WA 99164-2814
}

(Dated: November 2, 2018)

\begin{abstract}
The sensitivity of a wave field's evolution to small perturbations is of fundamental interest. For chaotic systems, there are two distinct regimes of either exponential or Gaussian overlap decay in time. We develop a semiclassical approach for understanding both regimes, and give a simple expression for the crossover time between the regimes. The wave field's evolution is considerably more stable than the exponential instability of chaotic trajectories seem to suggest. The resolution of this paradox lies in the collective behavior of the appropriate set of trajectories. Results are given for the standard map.

PACS numbers: $05.45 . \mathrm{Mt}$, 03.65.Sq
\end{abstract}

There is a multitude of physical systems in which the quantum evolution of a particle or that of a wave field interacts with a time-varying, complicated, essentially-unknowable medium. Well known examples include acoustic waves propagating long ranges through the ocean's sound channel [1], starlight passing through our atmosphere [2], and electrons diffusing through a metal [3]. In a similar vein, wave/quantum evolution problems whose underlying ray/classical analogs are simple chaotic systems, may possess parameters which are tunable or time varying as well. In all of these systems, understanding the effects that system changes have on the dynamics is of great interest. In this letter, we investigate the problem of the overlap decay of two initially identical wave fields propagating in slightly different simple, chaotic systems and using semiclassical dynamics derive the functional dependence of the decay process on time, Planck's constant, and perturbation strength.

Recently, the overlap between time-evolved perturbed and unperturbed states has been studied in many contexts and guises. The overlap has been suggested as a measure for the stability of quantum motion 画. It was identified with the Loschmidt echo and studied in the context of polarization echoes in nuclear magnetic resonance [5, 6] where a cross-over between Gaussian and exponential behavior of the overlap was observed. In quantum computing, it is equivalent to the fidelity which measures the loss of phase coherence 7]. The fidelity was shown to have different functional decay forms in the chaotic and integrable limits $[8]$.

Over the past ten years, it has been shown that semiclassical theories of wave mechanics based on rays/trajectories are capable of quantitatively reproducing evolving wave fields in chaotic dynamical systems for significant propagation times during which the chaos can become extremely well developed [9, 10]. Extensions of these semiclassical methods should therefore provide an accurate theoretical approach for understanding overlap decay behavior to at least the same significant times. We therefore couple a semiclassical approach with classical and quantum perturbation theory as appropriate for the small system changes we are considering, and methods from random matrix theory, which are known to apply to fully chaotic systems.

A number of paradoxes arise in considering the semiclassical limit. For example, in a classically chaotic system a slight perturbation of the initial conditions causes an exponential divergence of the rays and their action differences; the exponent characterizing this divergence is the Lyapunov exponent. In addition, perturbing the Hamiltonian, but using the same initial conditions, also causes the rays to exponentially diverge with the same Lyapunov exponent as for a change in the initial conditions. Wave fields behave very differently under perturbations than rays. The same wave field propagated in two slightly different systems has an overlap which decays in time on a scale proportional to the square of the strength of the system changes. On the other hand, two wave fields starting from different initial conditions in the same system have an overlap which is constant in time. Those paradoxes that exist must have resolutions or the semiclassical theories could not be such faithful approximations. Their resolutions hold the promise of revealing new features of either classical or wave mechanics. We discuss one example at the end of this letter.

Consider an evolving quantum wave function $\left|\alpha(t)_{\lambda}\right\rangle$ where $\lambda$ represents parameters that define the system. The overlap decay is defined as:

$$
\mathcal{C}(\epsilon ; t)=\mathcal{C}_{\lambda}(\epsilon ; t)=\left|\left\langle\alpha\left|\hat{U}_{\lambda-\epsilon / 2}^{\dagger}(t) U_{\lambda+\epsilon / 2}(t)\right| \alpha\right\rangle\right|^{2}
$$

where $\epsilon$ serves as the small parameter; the use of $\pm \epsilon / 2$ reduces next-to-leading perturbative corrections. $\mathcal{C}(\epsilon ; t)$ can be conceptualized equally well the squared overlap of an initial state $|\alpha\rangle$ propagated forward in time with two slightly different Hamiltonians or as $|\alpha\rangle$ propagated forward in time with one Hamiltonian and then backward in time with a 
slightly different one. In the limit that the system is strongly chaotic, the statistical properties of $\mathcal{C}_{\lambda}(\epsilon ; t)$ would be stationary rendering $\lambda$ largely a superfluous subscript.

Assuming the Hamiltonian is differentiable with respect to $\lambda$, let $V=\partial H(\lambda) / \partial \lambda$. The simplest approximate expression for $\mathcal{C}(\epsilon ; t)$ follows by keeping only the leading quantum perturbation corrections to the energies since they are divided by $\hbar$; this is clearly insufficient to describe fully the overlap decay behavior, but it does describe the first limiting regime. The resulting expression is

$$
\left.\left.\mathcal{C}(\epsilon ; t) \approx\left|\sum_{n}\right| b_{\alpha n}\right|^{2} \exp \left(-i \epsilon V_{n n} t / \hbar\right)\right|^{2}
$$

where $b_{\alpha n}=\left\langle\alpha(0) \mid n_{\lambda}\right\rangle$ and $V_{n n}=\left\langle n_{\lambda}|V| n_{\lambda}\right\rangle$.

In the strong chaotic limit, Eq. (2), can be evaluated using statistical arguments. Random matrix theory asserts that the eigenvalues and eigenfunctions are uncorrelated, and thus the amplitudes and phases statistically decouple; semiclassical arguments have also been developed that support this approach. The phases are Gaussian random distributed and are proportional to the derivative of the eigenenergies with respect to the parameter, commonly called the level velocities. Thus, the sum can be replaced by an integral over the Gaussian density, which gives

$$
\mathcal{C}(\epsilon ; t) \approx \exp \left(-\epsilon^{2} \sigma_{E}^{2} t^{2} / \hbar^{2}\right)
$$

where $\sigma_{E}^{2}$ is the variance of the level velocities. In the small $\epsilon$ and strongly chaotic limit, the overlap decay has a Gaussian form with respect to time.

The level velocity variance is not a free parameter, and has been related to $V$ through a semiclassical approach 11 , 12 ; it is

$$
\sigma_{E}^{2} \approx \frac{g K(E)}{\pi \hbar \bar{d}}
$$

where $g$ is a symmetry index, $\bar{d}$ is the mean density of states, and $K(E)$ is the classical action diffusion constant given by $[13$

$$
K(E)=\int_{0}^{\infty}\langle V(\mathbf{p}(0), \mathbf{q}(0) ; \lambda) V(\mathbf{p}(t), \mathbf{q}(t) ; \lambda)\rangle_{p} d t
$$

The averaging is defined over the primitive periodic orbits of very large period.

Equations (3-5) turn out to describe a very restricted range of $\epsilon$ centered at zero. A semiclassical approach without the quantum perturbation limitations can be developed that is valid over a much broader range. The semiclassical construction of an evolving wave function begins with the propagator

$$
\begin{aligned}
\left\langle q^{\prime}|\hat{U}| q\right\rangle \approx & \left(\frac{1}{2 \pi i \hbar}\right)^{d / 2} \sum_{j}\left|\operatorname{Det}\left(\frac{\partial^{2} W_{j}\left(\mathbf{q}, \mathbf{q}^{\prime} ; t\right)}{\partial \mathbf{q} \partial \mathbf{q}^{\prime}}\right)\right|^{1 / 2} \\
& \times \exp \left(i W_{j}\left(\mathbf{q}, \mathbf{q}^{\prime} ; t\right) / \hbar-\frac{i \pi \nu_{j}}{2}\right)
\end{aligned}
$$

The phase is specified by the time integral of the Lagrangian $W_{j}\left(\mathbf{q}, \mathbf{q}^{\prime} ; t\right)$ and an index based on the properties of the conjugate points (like focal points), $\nu_{j}$.

The overlap decay in terms of the propagator is

$$
\begin{aligned}
\mathcal{C}(\epsilon ; t)= & \mid \int \mathrm{d} q \mathrm{~d} q^{\prime} \mathrm{d} q^{\prime \prime}\langle\alpha \mid q\rangle\left\langle q\left|\hat{U}_{\lambda-\epsilon / 2}^{\dagger}(t)\right| q^{\prime}\right\rangle \\
& \times\left.\left\langle q^{\prime}\left|\hat{U}_{\lambda+\epsilon / 2}(t)\right| q^{\prime \prime}\right\rangle\left\langle q^{\prime \prime} \mid \alpha\right\rangle\right|^{2}
\end{aligned}
$$

For simplicity, we have chosen the initial states to be point sources, i.e. $\langle q \mid \alpha\rangle=\delta\left(q-q_{0}\right)$, but the following theory is generalizable to any initial state.

The most important contribution to the overlap decay is each orbit's action change due to its division by $\hbar$. Thus, changes in the amplitudes are ignored, so

$$
\begin{aligned}
\mathcal{C}(\epsilon ; t) \approx & \mid \frac{1}{(2 \pi \hbar)^{d}} \int \mathrm{d} q \sum_{n}\left(\frac{\partial^{2} W_{n}\left(q, q_{0} ; t\right)}{\partial q \partial q_{0}}\right) \\
& \times\left.\exp \left[\frac{i}{\hbar} \Delta W_{n}\left(q, q_{0} ; t\right)\right]\right|^{2}
\end{aligned}
$$



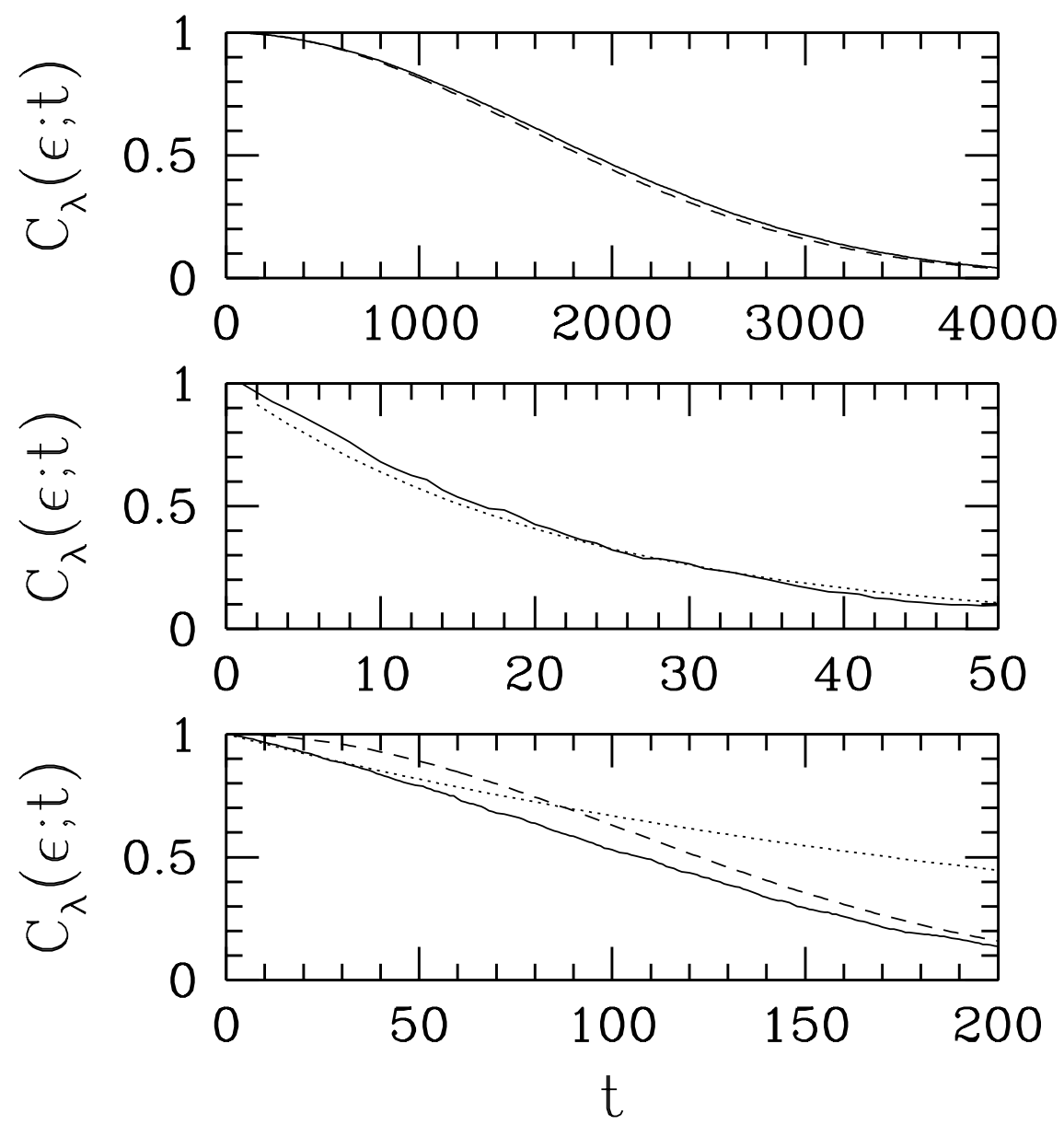

FIG. 1: Examples of Gaussian, exponential and cross-over decay behaviors. The quantum kicked rotor curves are solid, and the theoretical curves dashed. The parameters are $N=350, \lambda=18, g=4, K(E)=\left(1+2 J_{2}(\lambda)\right) / 4(2 \pi)^{4}$, and in $(\mathrm{a}) \epsilon=10^{-4}$, in (b) $5 * 10^{-3}$, and in (c) $1.5 * 10^{-3}$. Note that the equivalent expression of Eq. (勾) for maps is $\sigma_{E}^{2}=2 g K(E) / N$.

The $\Delta W_{n}\left(q, q_{0} ; t\right)$ are the action differences of two rays that begin at $q_{0}$ and end at position $q$ in a time $t$ and are continuously deformable into each other; bifurcations are neglected. First order classical perturbation theory gives

$$
\Delta W_{n}\left(q^{\prime}, q ; t\right)=\epsilon \int_{0}^{t} \frac{\partial L_{n}\left(q^{\prime}, q ; t^{\prime}\right)}{\partial \lambda} d t^{\prime}
$$

where the amplitude in Eq. (8) and the Lagrangian are evaluated along the orbits of $H(\lambda)$. In general, stationary phase integration cannot be performed on Eq. (8) because the action differences are less than $\hbar$.

In strongly chaotic systems or random media, the classical action differences for long orbits obey a central limit theorem. The contributions of the derivative of the Lagrangian along parts of an orbit separated widely in time are uncorrelated. Furthermore, each orbit has a unique history in which it visits the available phase space. Thus, the changes in action will be Gaussian distributed for sufficiently long times. The integral over $q$ can be replaced by a Gaussian probability integral over the action change which gives

$$
\mathcal{C}(\epsilon ; t) \approx \exp \left(-\sigma_{W}^{2} / \hbar^{2}\right)
$$

$\sigma_{W}^{2}$ is the variance of the action differences and is related to the same classical diffusion constant $K(E)$ as are the level velocities 13],

$$
\sigma_{W}^{2}=2 \epsilon^{2} K(E) t
$$



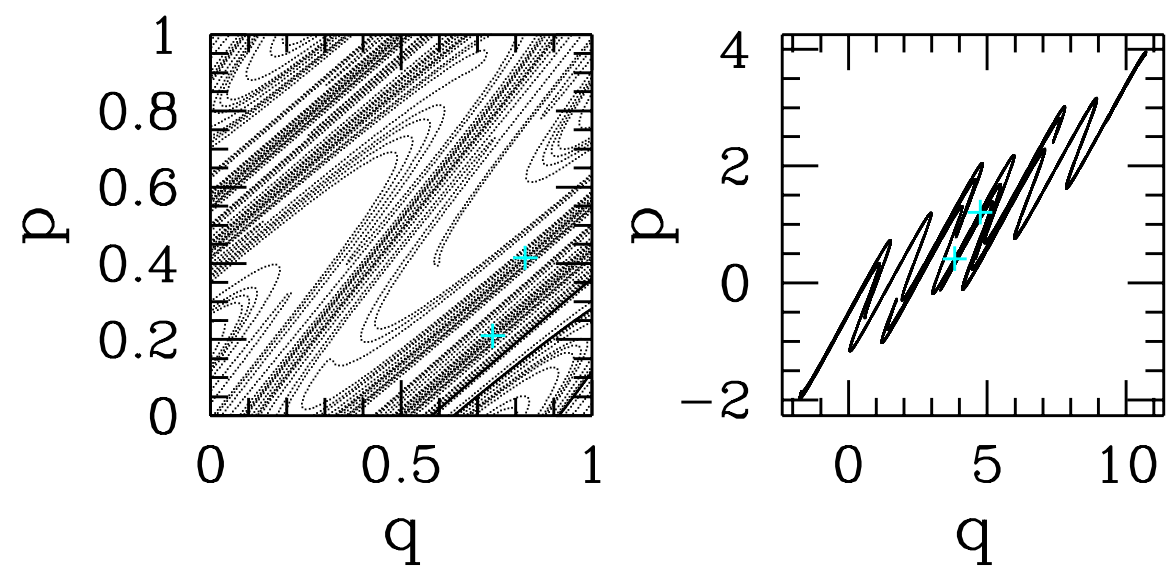

FIG. 2: The same two initial manifolds $\left(q_{0}=0.6\right)$ propagated four time steps through two slightly different media in the quantum kicked rotor where $\lambda_{1}=6.0$ and $\lambda_{2}=6.02$. The left panel is on the torus and the right panel is unfolded in phase space.

In contrast to the quantum perturbative argument giving a quadratic $t$-dependence in the exponential, here the argument is linear.

The exponential or Gaussian dependence dominates over the time range in which the argument of the exponential takes on the lesser value. The cross-over between the two regimes occurs when their arguments are equal. Simple algebra gives the cross-over time scale $t^{*}=h \bar{d} / g$, which is essentially the Heisenberg time, $\tau_{H}$. Exponential decay dominates if the decay is completed by $\tau_{H}$, and Gaussian decay dominates if little decay has occurred by $\tau_{H}$; otherwise the decay has components of both behaviors. In terms of the parameters, the question is

$$
\epsilon^{2} \stackrel{?}{<} \frac{\hbar^{2}}{2 K(E) \tau_{H}}=\frac{\hbar g}{4 \pi \bar{d} K(E)}
$$

This cross-over fits naturally with the approximations made in deriving the exponential and Gaussian behaviors. The classical action correlations necessary for a semiclassical theory to "know" the spectrum is discrete were not included whereas the simplified quantum perturbation theory left out coupling with neighboring levels which would be completely dephased by $\tau_{H}$.

We use the standard map, a paradigm of chaos for large kicking strength, to illustrate our main results. The classical map is defined by

$$
\begin{aligned}
p_{i+1} & =p_{i}-(\lambda / 2 \pi) \sin \left(2 \pi q_{i}\right) \bmod (1) \\
q_{i+1} & =q_{i}+p_{i+1} \bmod (1)
\end{aligned}
$$

For $\lambda$ greater than 6 or so, the system is strongly chaotic. The quantized propagator with $N$ discrete levels is given by

$$
\begin{aligned}
\left\langle n^{\prime}|\hat{U}| n\right\rangle= & \frac{1}{\sqrt{i N}} \exp \left[i \pi\left(n-n^{\prime}\right)^{2} / N\right] \\
& \times \exp \left(i \frac{k N}{2 \pi} \cos \left[2 \pi\left(n^{\prime}+a\right) / N\right]\right)
\end{aligned}
$$

where $n, n^{\prime}=0, \ldots, N-1$ and $a$ is a phase term which we set equal to zero. The effective Planck constant is given by $h=1 / N$.

In Fig. 边, we show three example overlap decays. The upper panel illustrates an example of Gaussian decay, the middle panel illustrates exponential decay, and the bottom panel illustrates a case where the cross-over between the two cases can be seen. The theoretical curves drawn for comparison have the analytic value of $K(E)$ 11 inserted, and so involve no parameter fitting whatsoever. The agreement is excellent.

At first consideration, it may seem hopeless to apply a first order classical perturbation theory to a chaotic system. The action difference cannot grow faster than the time, whereas the classical action of a trajectory associated with 

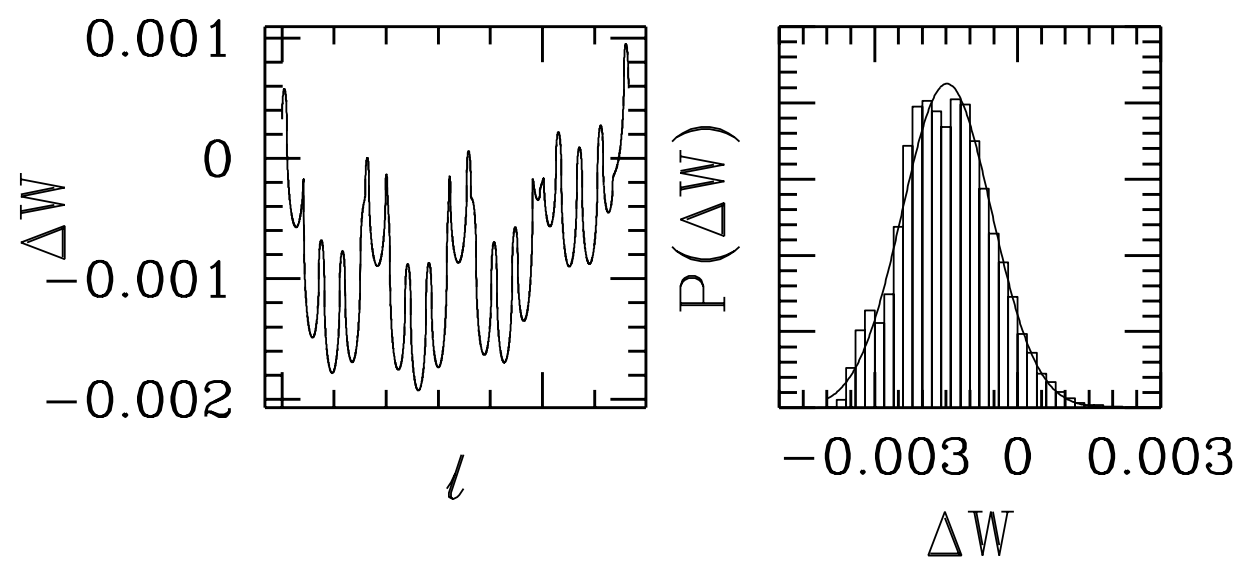

FIG. 3: The action changes along the manifold. The solid line in the the left panel is the exact action differences for the two manifolds in Fig. 2 and the dashed line is the perturbation result. The right panel is a histogram of the action changes for eight time steps of the initial manifolds in the right panel and the solid line is a Gaussian fit to the histogram.

a particular initial condition deviates exponentially. At best, the first order approximation should be valid no longer than a logarithmically short time scale, $\tau^{*}=-\frac{1}{\lambda} \ln \epsilon$, and the theory given above should not work. The resolution rests in considering the difference between the behavior of a single trajectory and the collection of trajectories underlying the construction of a wave field. In time-dependent WKB theory, the collection is a Lagrangian manifold. In our example here with a position state initial condition, the initial manifold is just the set of initial conditions containing all momenta and $q=q_{0}$. To propagate the state, the manifold is propagated. Likewise to distinguish the propagation of the same initial wave function for slightly different initial conditions entails propagating the manifold under the two slightly different dynamics. This is illustrated in the left panel of Fig. 2 for an initial $q$-manifold. The two manifolds are so close as to be nearly indistinguishable. In the bottom panel where the exact same propagated manifolds are depicted except with the phase space torus unfolded into a plane, the +'s mark the final point of a single initial condition. The final point deviates an entire unit cell away, and yet has only essentially slid along the original manifold. The key feature of the slowly varying manifold is related to the changing locations of the folding points, which generally are associated with caustics. The manifold has a strong structural stability that still allows for exponential sensitivity to perturbations for its individual members.

Thus, the construction of an evolving wave function reflects the stability of the manifold, not the individual trajectories. For example, in the Green function, the condition of starting at $q^{\prime}$ and ending at $q$ naturally selects trajectories, continuously deformable into each other, whose action differences under perturbation are minimal. The first order classical perturbation theory accounts for this effect. In Fig. 3, the action difference is plotted in the left panel as a function of position along the manifold. The first order classical perturbation prediction is superposed, and it is impossible to distinguish them; it is extremely accurate. It turns out that the extrema of the action changes correspond to the points where the two manifolds intersect. In the right panel, we see that the fluctuations are such as to give a good approximation to a Gaussian density. In the limit of longer orbits, the results would get better and better.

In conclusion, the overlap of a wave function evolving under two slightly different chaotic dynamics decays either exponentially or in a Gaussian fashion. The decay scales and cross-over behavior can be derived semiclassically. We have not shown it here, but deviations due to not being 'chaotic enough' or other statistical shortcomings are captured by the semiclassical theory. However, the correlations and deviations are buried in the orbit sums, and not easily extracted other than by numerical summation. An interesting consequence of classical mechanics is that manifolds are much more stable against perturbation than the trajectories of which they are comprised. First order classical perturbation theory yields an excellent approximation to the changes of the actions, and a statistical theory of these action variations leads to a very good agreement of the overlaps. The stability of wave fields is connected to the manifolds and the associated phases, and not the individual trajectories.

We gratefully acknowledge important discussions with M. A. Wolfson and T. Prosen, and support from the Office of Naval Research under Grant No. NSF-PHY-0098027 and the National Science Foundation under Grant No. N0001498-1-0079.

Note: After this work was completed, we discovered Ref. 114 which also examines the overlap decay. 
[1] S. M. Flatté, R. Dashen, W. H. Munk, and F. Zachariasen, Sound Transmission through a Fluctuating Ocean, (Cambridge University Press, Cambridge, 1979).

[2] M. V. Berry, J. Phys. A: Math. Gen. 10, 2061 (1977).

[3] B. L. Al'tshuler and B. Z. Spivak, Pis'ma Zh. Eksp. Teor. Fiz. 42, 294 (1985) [JETP Lett. 42, 447 (1985)]; S. Feng, P. A. Lee and A. D. Stone, Phys. Rev. Lett. 56, 1960 (1986).

[4] A. Peres, Quantum Theory: Concepts and Methods, (Kluwer AP, 1995).

[5] H. M Pastawski, P. R. Levstein, and G. Usaj, Phys. Rev. Lett. 75, 4310 (1995); G. Usaj, H. M Pastawski, and P. R. Levstein, Mol. Phys. 95, 1229 (1998).

[6] R. A. Jalabert and H. M. Pastawski, Phys. Rev. Lett. 86, 2490 (2001).

[7] M. A. Nielsen and I. L. Chuang, Quantum Computation and Quantum Information, (Cambridge University Press, 2000).

[8] T. Prosen, quant-ph/0106149.

[9] S. Tomsovic and E. J. Heller, Phys. Rev. Lett. 67, 664 (1991); Phys. Rev. E 47, 282 (1993); P. W. O'Connor, S. Tomsovic, and E. J. Heller, Physica D 55, 340 (1992); M.-A. Sepúlveda, S. Tomsovic and E. J. Heller, Phys. Rev. Lett. 69, 402 (1992).

[10] F. Mortessagne, O. Legrand and D. Sornette, Europhys. Lett. 33, 417 (1996); M. G. Brown and M. A. Wolfson, private communication.

[11] N. R. Cerruti, A. Lakshminarayan, J. H. Lefebvre, and S. Tomsovic, Phys. Rev. E 63, 016208 (2000); A. Lakshminarayan, N. R. Cerruti, and S. Tomsovic, Phys. Rev. E 60, 3992 (1999); erratum: Phys. Rev. E 61, 6031 (2000).

[12] M. V. Berry and J. P. Keating, J. Phys. A: Math. Gen. 27, 6167 (1994).

[13] O. Bohigas, M. J. Giannoni, A. M. Ozorio de Almeida and C. Schmit, Nonlinearity 8, 203 (1995).

[14] Ph. Jacquod, P. G. Silvestrov and C. W. J. Beenakker, nlin.CD/0107044. 